\title{
Gestión de crisis e imagen de marca de las organizaciones. Cómo desarrollar un plan de actuación
}

\section{Issues management \& corporate brand image. How to develop an action plan}

PALOMA SANZ-MARCOS

ORCID: http://orcid.org/0000-0002-6103-6993

Universidad de Sevilla

Departamento de Comunicación

audiovisual y publicidad

palomasanz@us.es

Fecha de recepción: 28/10/2019

Fecha de aceptación: 4/11/2019

DOI: http://dx.doi.org/10.12795/9788447221912.069 Pp.: 1592-1614 
Esta comunicación tiene como objetivo realizar un plan de trabajo de mejora e innovación docente en la asignatura Teoría y Estructura de las RR.PP (10 de Grado en Publicidad y RR.PP). A través de una metodología de Aprendizaje Basado en Problemas (ABP) y Estudio de Casos se plantea la posibilidad de que los alumnos aprendan parte de los contenidos teóricos de la asignatura a través de la resolución de un problema real que relaciona los contenidos del temario. Es por ello que el objetivo fundamental que se persigue es potenciar el aprendizaje significativo del alumno mediante la puesta en marcha de un plan de actividades que el propio alumnado tendrá que resolver con objeto de preservar la imagen corporativa de una empresa. De este modo, el proyecto contempla un desarrollo autónomo de los conocimientos por parte del alumnado, al mismo tiempo que propone una metodología aplicada al mundo real y de gran interés para su formación.

Palabras clave: Teoría y estructura de las RR.PP, Grado en Publicidad y RR.PP, Docencia Universitaria, Experimentación docente universitaria, Aprendizaje Basado en Problemas.

\section{Abstract}

This communication aims to make a work plan of improvement and teaching innovation in the subject Theory and Structure of the RR.PP (1으 of Degree in Advertising and RR.PP). Through a methodology based on problem-based learning (PBL) and a Case Study, it is proposed to make that students learn part of the theoretical contents of the subject through the resolution of a real problem that relates the contents of the syllabus. That is why the fundamental objective pursued is to enhance the significant learning of the student through the implementation of a plan of activities that the students themselves will have to solve in order to preserve the corporate image of a company. In this way, the project contemplates an autonomous development of the knowledge by the students, at the same time it proposes a methodology applied to the real world and of great interest for its formation.

Key words: Theory and Structure of PR, Advertising and PR Degree, University Teaching, University Teaching Innovation, Problem Based Learning (PBL). 


\section{Descripción del contexto de la asignatura}

La asignatura Teoría y Estructura de las RR.PP pertenece al primer curso del grado en Publicidad y RR.PP y se compone de 6 créditos de carácter obligatorio. Este Ciclo de Mejora en el Aula (CIMA, en adelante) se aplica a estudiantes del centro adscrito a la Universidad de Sevilla EUSA. Las características del alumnado son, por lo general, estudiantes que comienzan por primera vez su experiencia universitaria y se reparten en una clase de 64 alumnos. No obstante, dada la gran cantidad de matriculados, la asignatura sufre un desdoble resultando en dos grupos de unos 32 alumnos. Es importante destacar que, dado que se trata de una de las pocas asignaturas dedicadas a las RR.PP durante toda la carrera, suele contar con el entusiasmo y motivación del alumnado. En este sentido, esta asignatura supondrá un punto de partida muy útil para manejar la información acerca de las RR.PP a lo largo de toda la carrera. Las actividades aquí propuestas pretenden instalar en el alumno herramientas para manejar la imagen de una empresa y enfocar sus posibles estrategias.

\section{Diseño previo del CIMA}

El diseño de este CIMA se inicia con el planteamiento de los conceptos a desarrollar en el aula para que el alumnado tenga una visión general de lo que verá a lo largo de esta parte de la asignatura. Dado que se trata de la primera parte del temario, como primer paso se ha realizado un mapa de contenidos. Este mapa viene a clarificar y ordenar toda la materia que se empleará durante el citado ciclo. Su objetivo es describir el aprendizaje deseable esperado por la docente para alcanzar los conocimientos necesarios para accionar un plan de crisis en RR.PP. Concretamente, la temática en cuestión se enfoca 
en la necesidad de controlar el tiempo e identificar a lo públicos afectados para llevar a cabo un correcto plan de actuación (Wilcox, et al., 2006). Se trata de un bloque fundamental para conocer en profundidad la importancia de los públicos y la imagen de las empresas para las relaciones públicas. Si bien se trata de una temática compleja, introduce de manera apropiada el sentido que tiene la profesión de los relacionistas públicos y su importancia en las organizaciones. Como se puede observar en la figura 1, el ciclo se desarrollará en torno a un gran problema que parte de una pregunta: ¿Cómo se gestiona una crisis de imagen corporativa? Para tratar de resolver este gran problema, se plantean tres grandes preguntas que bien podrían entenderse como mini problemas que tratan de aportar soluciones al gran problema inicial. Cabe destacar que estas preguntas mantienen una estrecha relación entre sí. En este sentido, la interacción entre los contenidos se hace esencial dado que trata de ofrecer una interrelación que se supone óptima desde el punto de vista psicológico del alumno (Porlán, 2017: 60) para que éste adquiera un aprendizaje significativo.

Jornadas de Formación e Innovación Docente del Profesorado | № 2 (2019) Esta obra se distribuye con la licencia Creative Commons 
PALOMA SANZ-MARCOS

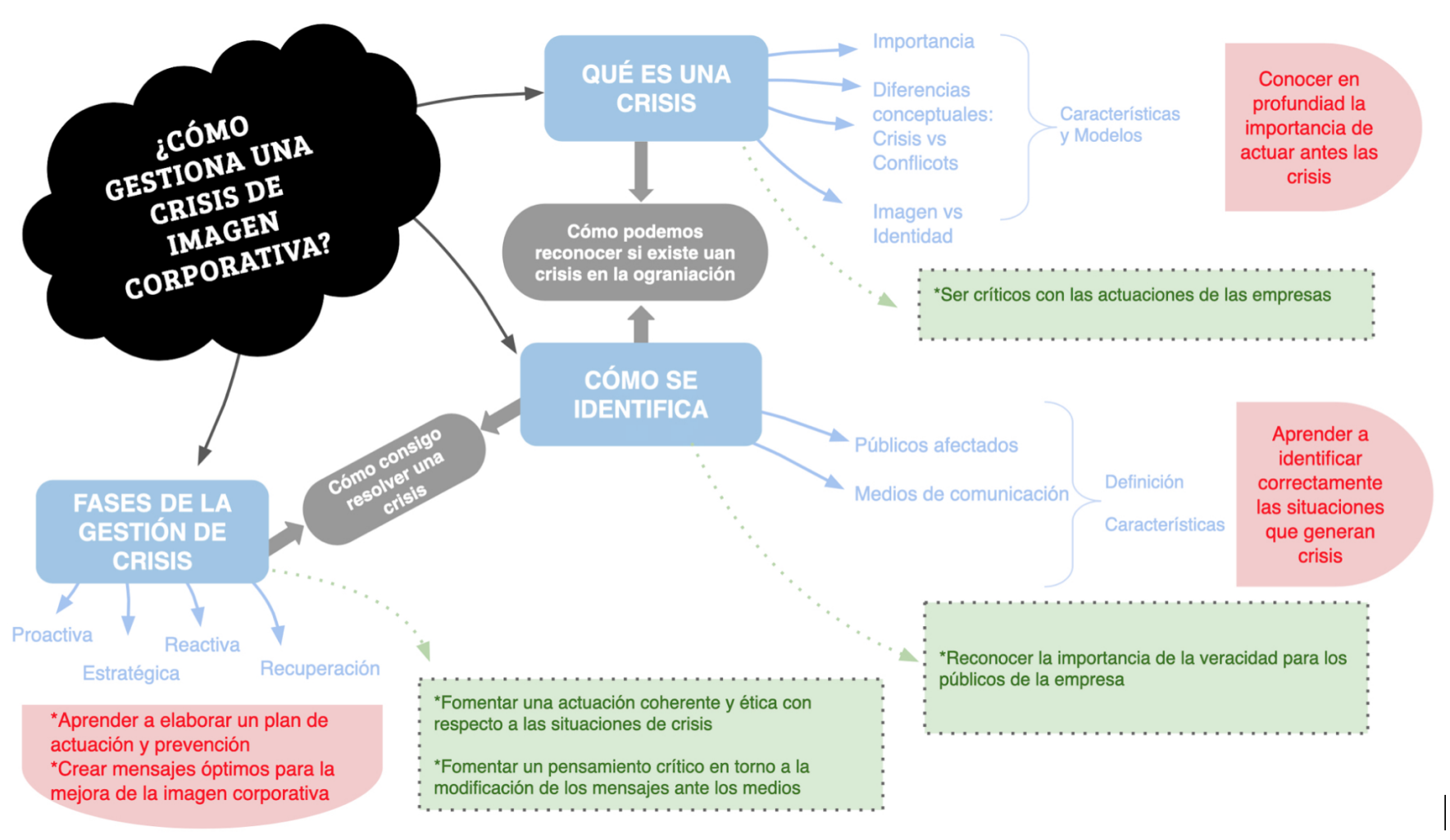

Figura 1. Mapa de contenidos conceptuales, procedimentales y actitudinales

Jornadas de Formación e Innovación Docente del Profesorado I № 2 (2019) Esta obra se distribuye con la licencia Creative Commons Reconocimiento-NoComercial-SinObraDerivada Internacional (CC BY-NC-ND 4.0.) 
El mapa consta de tres grandes bloques, en azul, vemos los contenidos de tipo conceptual. Estos contenidos son los que normalmente se imparten de manera magistral clásica, es decir, basado en un modelo metodológico fundamentalmente transmisivo. Como ejemplo de este tipo de contenido, encontramos aquel dedicado a conocer qué es exactamente una crisis y por qué es necesario que comprendamos sus consecuencias como futuros relacionistas públicos. Estos conceptos son fundamentalmente de tipo teórico y se tratarán de complementar a través de preguntas y actividades que se relacionen e intercalen con un contenido y metodología más denso y teórico. Lo más significativo para este CIMA es que para empezar con este contenido se planteará un problema real del que partiremos para aplicar la información de corte teórica.

Luego, en rojo, tendríamos los contenidos de tipo procedimental, los cuales vienen a poner en práctica los contenidos conceptuales. Ejemplo de ello encontramos el planteamiento de actividades como conocer la importancia de una crisis a través del conocimiento de experiencias anteriores de otras empresas, elaborar un plan de actuación y recuperación, etc., que se habrán de llevar a cabo a través de actividades prácticas grupales. Luego, en verde, encontraríamos los contenidos de tipo actitudinal. Estos tratan de estimular el sentido crítico de los alumnos a partir de los conceptos que se ven en la asignatura. De alguna manera, el objetivo de estos conceptos es reflexionar en torno al ámbito deontológico de la profesión del relacionista público. Concretamente, para este tipo de contenido se hace muy necesario fomentar este tipo de criterio y perspectivas debido a la importancia capital que tiene para la gestión de las crisis empresariales.

Jornadas de Formación e Innovación Docente del Profesorado | № 2 (2019) Esta obra se distribuye con la licencia Creative Commons 


\section{Modificaciones introducidas en el CIMA del curso 2019-2020}

Dado que para esta asignatura ya se implementó un CIMA en el curso 2018-2019, se ha procurado introducir una serie de mejoras que provienen de las reflexiones recogidas en el ciclo anterior. En primer lugar, uno de los principales anhelos con respecto al curso pasado fue la necesidad de hacer una mejor gestión del tiempo. A pesar de que la experiencia fue muy positiva, el alumnado manifestó cierto agobio a la hora de realizar las actividades prácticas vistas en clase. Con objeto de evitar el desgaste del alumnado se ha previsto la elaboración de un documento que contenga las instrucciones detalladas del proceso a llevar a cabo en cada tarea propuesta. Este documento, si bien no descarga el trabajo del alumno, sí ofrecerá un buen punto de partida para que el grupo pueda organizar con tiempo todas las tareas que tendrá que llevar a cabo y saber con antelación el procedimiento y tiempo de cada una de ellas. Asimismo, en el grueso de estas actividades se contemplan tareas para realizar en casa. Algo que en el anterior ciclo no se tuvo en cuenta.

Otra de las objeciones que se plantearon en el curso pasado fue la de realizar algún cambio en cuanto a los contenidos. Dado que hubo mucha carga teórica, se reflexionó acerca de la posibilidad de presentar la materia a través del visionado de documentales o vídeos. Si bien para este ciclo el tema elegido es completamente diferente, es cierto que a la hora de elaborar la dinámica de clase se ha procurado presentar el contenido de una manera innovadora. En este caso, se ha acudido a una elaboración del material más autónoma por parte del alumnado. Como se observará más adelante, se llevarán a cabo actividades que pondrán en práctica la teoría vista en clase en tiempo real, en este sentido, muchas de estas actividades contemplan la búsqueda de material bibliográfico por parte de los alumnos, la adaptación de ese material al trabajo de clase y su consecuente presentación al resto de la clase con objeto de hacer una corrección global. 


\section{Cuestionario inicial-final}

Para valorar el estado de la clase con respecto a este mapa conceptual, es decir, para conocer un poco qué dominio tiene la clase en torno a estos conceptos, hemos diseñado un cuestionario inicial que será la primera "actividad" que le plantearemos a los alumnos. Este cuestionario estaría compuesto por 5 preguntas que parten directamente de los 3 grandes bloques conceptuales que se piensan tratar durante el ciclo de mejora.

\section{CUESTIONARIO INICIAL DE 3 CIMA - ASIGNATURA TEORÍA Y ESTRUCTURA DE LAS RR.PP}

1. ¿Crees que las empresas pueden verse afectadas por lo que los medios de comunicación difunden sobre ellas? Justifica tu respuesta, da argumentos.

2. ¿Qué entiendes por el concepto "Imagen corporativa"?

3. ¿Crees que si la imagen de una empresa se ve afectada negativamente por lo que los medios de comunicación difunden sobre ella esto afectaría solo a su público objetivo?, ¿Podrían verse afectados otros públicos no tan directos?

4. ¿Es posible que las empresas detecten con antelación posibles crisis?

5. ¿Crees que las empresas pueden recuperarse de una crisis?

Las preguntas parten de estos bloques y tratan de no plantear directamente los contenidos que se verán en clase. Se trata de conocer qué conocimientos previos tienen los alumnos para después de aplicar el ciclo, saber qué han aprendido. Este mismo cuestionario inicial se planteará cuando hayan acabado todas las actividades teóricas y prácticas para hacer un seguimiento y comprobación de lo aprendido por el alumnado. 
Modelo metodológico posible

Asimismo, para poner en práctica este mapa conceptual, se ha pensado en un modelo metodológico posible que logre alcanzar el aprendizaje de la materia.

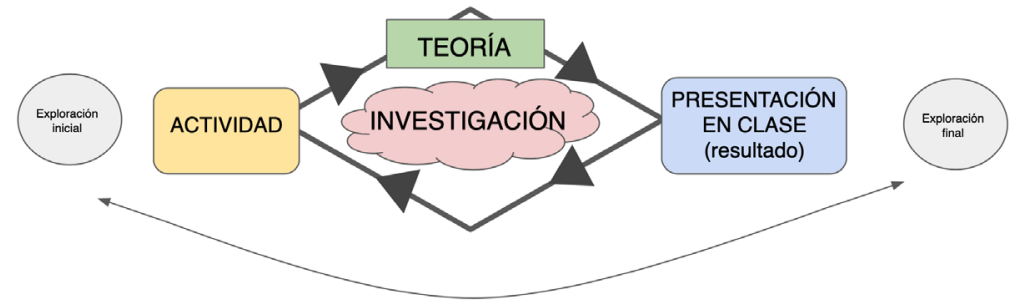

Figura 2. Modelo metodológico posible

Como se observa en la figura 2, este modelo metodológico comprende la investigación como el eje del aprendizaje. Dado que uno de los principales objetivos que se plantea en este CIMA es que el alumnado adquiera el conocimiento de la manera más autónoma posible, se entiende que la indagación y el descubrimiento ocupan un lugar destacado en el proceso. En este sentido, se ha pretendido proponer un alejamiento de aquellos modelos de enseñanza más cercanos al transmisivo en el que el papel de la teoría y el profesor son más destacables que el del alumnado. No obstante, la teoría sigue teniendo un papel clave en el aprendizaje. Este modelo contempla que la teoría y la investigación han de ir vinculados, es por ello que en la figura se encuentran cercanos. Asimismo, este modelo, trata de situar al alumno en el centro del aprendizaje a través de una metodología que parte de un problema que él mismo tiene que resolver. Cabe destacar que tanto el problema como algunas de las directrices introductorias, son indicadas por la profesora que funcionará como guía del proceso de aprendizaje. De este modo, se pretende hacer una especie de hibridación entre lo que plantea Bain (2004) a través de sus talleres y seminarios y Finkel (2008) con su estilo de dar clase con la boca cerrada. 
Tanto el cuestionario inicial como el final, están reflejados en el esquema visual a través de la exploración inicial y final. Se considera que ambas son necesarias para el correcto desarrollo de este modelo en tanto a que producen un contenido informativo muy útil para la profesora. Así, el cuestionario inicial servirá como una herramienta para diagnosticar el estado de la clase y poder ajustar los contenidos diseñados de un modo mucho más personalizado. En cuanto al cuestionario final, se trata de una herramienta que visualiza de una manera muy ilustrativa el desarrollo y evolución que han seguido los alumnos, algo, sin duda, de especial interés para la reflexión del trabajo de la profesora. No obstante, y atendiendo al CIMA anterior, se trata de una actividad que suele entusiasmar al alumnado debido a que identifican con claridad lo aprendido durante la clase.

El proyecto en cuestión trata sobre cómo gestionar una crisis de imagen corporativa de una empresa. Concretamente, se tomará como referencia un caso real que le ocurrió a la firma DODOT. Se trata de una noticia que afectó significativamente a la firma y que habría que resolver para volver a ganar la confianza de los consumidores. Esto ofrece una magnífica oportunidad para poner en evidencia la dificil relación que se puede establecer entre una empresa y los medios de comunicación. La idea es identificar la importancia de la crisis para los públicos y diseñar un plan de crisis para gestionar lo ocurrido. Como actividad final se planteará una exposición oral por grupos ante toda la clase con las acciones diseñadas para tratar de resolver el problema. No obstante, y con objeto de afianzar los conocimientos, se plantearán una serie de actividades prácticas anteriores. La primera de ellas, combinada con la exposición de una serie de contenidos de manera magistral, consistirá en la recopilación de información por parte del alumnado de noticias que se consideren potencialmente negativas para las empresas. El objetivo es buscar información por grupos para luego ordenarlas en una 
herramienta similar a una escalera de aprendizaje. Esta escalera pretende funcionar como una herramienta clarificadora de los materiales encontrados. Se tratará de ordenar las noticias por temática y por grado de relación con respecto a lo visto en clase. El alumno deberá de organizar la información en función de grandes grupos ateniendo a la teoría. El objetivo es conseguir que el alumno reflexione sobre la información encontrada. De este modo, no sólo se pretende hacer un buen uso de las herramientas de investigación y la lectura de medios de comunicación, al mismo tiempo y gracias a la elaboración de la escalera, se conseguirá establecer una relación y clasificación de los diferentes conceptos fundamentales como el de crisis o conflicto. Otra de las actividades que complementarán a la presentación final será la elaboración de un dossier de medios. Este dossier pretende ser un documento de gran utilidad para llevar a cabo la presentación final. Se trata de elaborar un documento en el que el alumno tiene que recopilar información de los medios de comunicación (en este caso repartiremos los medios según los diferentes grupos, de esta manera, unos se encargarán de los medios escritos, otros de los radiofónicos, etc,.) con objeto de identificar aquellos medios que tienen presencia en España y con los que un relacionista público se tendrá que relacionar para resolver crisis como la propuesta en el caso de la práctica final.

En definitiva este modelo pretende contribuir a un aprendizaje útil y significativo para el alumno, y que en el futuro pueda aplicar en futuras agencias de relaciones públicas. Asimismo, se propone presentar los contenidos de un modo interesante y atractivo tanto para el alumnado como para la profesora. 


\section{Secuencia de actividades}

En cuanto al diseño detallado de las actividades indicando para cada actividad, se ha pensado en el siguiente esquema que propone la temporalización y secuenciación de las actividades.

Tabla 1. Secuencia de actividades del CIMA.

\begin{tabular}{|c|c|c|c|c|}
\hline \multicolumn{5}{|c|}{ Dia 1(2h) } \\
\hline $\begin{array}{c}\text { Pregunta } \\
\text { relativa al mapa } \\
\text { de contenidos }\end{array}$ & - & - & $\begin{array}{l}\text { Qué es una } \\
\text { crisis }\end{array}$ & \\
\hline $\begin{array}{c}\text { Tipo de } \\
\text { actividad }\end{array}$ & Calentamiento inicial & Cuestionario inicial & Clase magistral & $\begin{array}{l}\text { Resolución } \\
\text { de dudas y } \\
\text { recapitulación } \\
\text { de la materia }\end{array}$ \\
\hline $\begin{array}{c}\text { Referencia } \\
\text { al modelo } \\
\text { metodológico }\end{array}$ & - & & TEORIA & \\
\hline Descripción & $\begin{array}{l}\text { Se saluda, recapitula } \\
\text { lo del día anterior, se } \\
\text { prepara la clase para } \\
\text { comenzar el silencio } \\
\text { y la clase } \\
\text { (10 minutos) }\end{array}$ & $\begin{array}{l}\text { Se advierte de que se } \\
\text { va a trabajar de un } \\
\text { modo diferente las } \\
\text { siguientes semanas y } \\
\text { se pasa el cuestionario } \\
\text { (20 minutos) }\end{array}$ & $\begin{array}{l}\text { Se introduce } \\
\text { el contenido } \\
\text { teórico de la } \\
\text { clase. Conceptos } \\
\text { como crisis, } \\
\text { gestión de } \\
\text { conflictos } \\
\text { o imagen } \\
\text { corporativa son } \\
\text { explicados por } \\
\text { la profesora } \\
\text { (50 min) }\end{array}$ & $\begin{array}{l}\text { Se deja un } \\
\text { tiempo para } \\
\text { volver a repasar } \\
\text { la teoría y se } \\
\text { plantearán } \\
\text { las posibles } \\
\text { dudas. Se cierra } \\
\text { explicando } \\
\text { la próxima } \\
\text { actividad } \\
\text { relacionada con } \\
\text { la escalera } \\
\text { ( } 40 \text { min) }\end{array}$ \\
\hline \multicolumn{5}{|c|}{ Dia 2 (2h) } \\
\hline $\begin{array}{l}\text { Pregunta } \\
\text { relativa al mapa } \\
\text { de contenidos }\end{array}$ & - & $\begin{array}{c}\text { Qué es una crisis } \\
\text { (puesta en práctica del } \\
\text { alumnado) }\end{array}$ & & \\
\hline $\begin{array}{c}\text { Tipo de } \\
\text { actividad }\end{array}$ & Calentamiento inicial & $\begin{array}{c}\text { Realización de la } \\
\text { actividad por parte del } \\
\text { alumnado }\end{array}$ & & $\begin{array}{c}\text { Cierre y } \\
\text { recapitulación } \\
\text { de lo aprendido }\end{array}$ \\
\hline
\end{tabular}

Jornadas de Formación e Innovación Docente del Profesorado | № 2 (2019)

Esta obra se distribuye con la licencia Creative Commons

Reconocimiento-NoComercial-SinObraDerivada

Internacional (CC BY-NC-ND 4.0.) 


\begin{tabular}{|c|c|c|c|c|}
\hline $\begin{array}{c}\text { Referencia } \\
\text { al modelo } \\
\text { metodológico }\end{array}$ & - & ACTIVIDAD & ACTIVIDAD & \\
\hline Descripción & $\begin{array}{l}\text { Se saluda, recapitula } \\
\text { lo del día anterior, se } \\
\text { prepara la clase para } \\
\text { comenzar el silencio } \\
\text { y la clase } \\
\text { (15 min) }\end{array}$ & $\begin{array}{c}\text { Se inicia la actividad } \\
\text { de búsqueda posibles } \\
\text { noticias que estén } \\
\text { relacionadas con el } \\
\text { contenido visto en } \\
\text { clase } \\
\text { (40 min) }\end{array}$ & $\begin{array}{c}\text { Se elabora } \\
\text { la escalera y } \\
\text { se sitúan las } \\
\text { noticias en cada } \\
\text { escalón a juicio } \\
\text { del alumnado } \\
\text { son explicados } \\
\text { por la profesora } \\
\text { (40 min) }\end{array}$ & $\begin{array}{c}\text { Se hace una } \\
\text { puesta en } \\
\text { común de todas } \\
\text { las noticias y se } \\
\text { hace hincapié } \\
\text { en la escalera } \\
\text { de cada grupo. } \\
\text { Se cierra } \\
\text { tratando de } \\
\text { consensuar una } \\
\text { escalera para } \\
\text { todos que esté } \\
\text { estrechamente } \\
\text { relacionada con } \\
\text { los conceptos } \\
\text { vistos en clase } \\
\text { (25 min) }\end{array}$ \\
\hline \multicolumn{5}{|c|}{ Día 3 (3h) } \\
\hline $\begin{array}{c}\text { Pregunta } \\
\text { relativa al mapa } \\
\text { de contenidos }\end{array}$ & - & $\begin{array}{c}\text { Cómo se identifica una } \\
\text { crisis }\end{array}$ & - & \\
\hline $\begin{array}{l}\text { Tipo de } \\
\text { actividad }\end{array}$ & Calentamiento inicial & $\begin{array}{c}\text { Investigación por parte } \\
\text { del alumnado }\end{array}$ & & \\
\hline $\begin{array}{c}\text { Referencia } \\
\text { al modelo } \\
\text { metodológico }\end{array}$ & & TEORÍA & INVESTIGACIÓN & \\
\hline Descripción & $\begin{array}{c}\text { Se saluda, recapitula } \\
\text { lo del día anterior, se } \\
\text { prepara la clase para } \\
\text { comenzar el silencio } \\
\text { y la clase } \\
\text { (10 min) }\end{array}$ & $\begin{array}{l}\text { Conectando con las } \\
\text { escaleras del día } \\
\text { anterior, se presenta } \\
\text { el caso real de DODOT } \\
\text { y se continúa con la } \\
\text { teoría prevista acerca } \\
\text { de la identificación de } \\
\text { las crisis } \\
\text { (45 min) }\end{array}$ & $\begin{array}{l}\text { Se plantea } \\
\text { la actividad } \\
\text { relacionada } \\
\text { con el dossier } \\
\text { que tendrán } \\
\text { que elaborar } \\
\text { los alumnos. Se } \\
\text { emplea el resto } \\
\text { de la clase en la } \\
\text { investigación del } \\
\text { problema } \\
\text { (65 min) }\end{array}$ & \\
\hline & & Día 4 (2h) & & \\
\hline
\end{tabular}

Jornadas de Formación e Innovación Docente del Profesorado I № 2 (2019) 


\begin{tabular}{|c|c|c|c|}
\hline $\begin{array}{l}\text { Pregunta } \\
\text { relativa al mapa } \\
\text { de contenidos }\end{array}$ & \multicolumn{3}{|c|}{-} \\
\hline $\begin{array}{l}\text { Tipo de } \\
\text { actividad }\end{array}$ & Calentamiento inicial & $\begin{array}{l}\text { Investigación por parte } \\
\text { del alumnado }\end{array}$ & $\begin{array}{l}\text { Cierre y fijación } \\
\text { de contenidos }\end{array}$ \\
\hline $\begin{array}{c}\text { Referencia } \\
\text { al modelo } \\
\text { metodológico }\end{array}$ & & TEORÍA & INVESTIGACIÓN \\
\hline Descripción & $\begin{array}{l}\text { Se saluda, recapitula } \\
\text { lo del día anterior, se } \\
\text { prepara la clase para } \\
\text { comenzar el silencio } \\
\text { y la clase } \\
\text { (10 min) }\end{array}$ & $\begin{array}{c}\text { Conectando con las } \\
\text { escaleras del día } \\
\text { anterior, se presenta } \\
\text { el caso real de DODOT } \\
\text { y se continúa con la } \\
\text { teoría prevista acerca } \\
\text { de la identificación de } \\
\text { las crisis } \\
\text { (45 min) }\end{array}$ & $\begin{array}{l}\text { Se plantea la actividad relacionada } \\
\text { con el dossier que tendrán que } \\
\text { elaborar los alumnos. Se emplea el } \\
\text { resto de la clase en la investigación } \\
\text { del problema } \\
\text { (65 min) }\end{array}$ \\
\hline \multicolumn{4}{|c|}{ Día 5 (2h) } \\
\hline $\begin{array}{l}\text { Pregunta } \\
\text { relativa al mapa } \\
\text { de contenidos }\end{array}$ & - & $\begin{array}{l}\text { Fases de gestión de } \\
\text { crisis }\end{array}$ & - \\
\hline $\begin{array}{l}\text { Tipo de } \\
\text { actividad }\end{array}$ & Calentamiento inicial & Clase magistral & $\begin{array}{c}\text { Trabajo autónomo por parte del } \\
\text { alumnado }\end{array}$ \\
\hline $\begin{array}{c}\text { Referencia } \\
\text { al modelo } \\
\text { metodológico }\end{array}$ & & TEORÍA & INVESTIGACIÓN \\
\hline Descripción & $\begin{array}{c}\text { Se saluda, recapitula } \\
\text { lo del día anterior, se } \\
\text { prepara la clase para } \\
\text { comenzar el silencio } \\
\text { de clase. Se advierte } \\
\text { de que estos últimos } \\
\text { días del cimA se } \\
\text { comenzará a trabajar } \\
\text { en el proyecto final } \\
\text { (15 min) }\end{array}$ & $\begin{array}{c}\text { Se introduce el } \\
\text { contenido teórico } \\
\text { de la clase. Los } \\
\text { conceptos a tratar son } \\
\text { la identificación de las } \\
\text { distintas fases de las } \\
\text { crisis } \\
(60 \mathrm{~min})\end{array}$ & $\begin{array}{l}\text { Los alumnos comienzan a trabajar } \\
\text { en el proyecto planteado. Hoy } \\
\text { es día de resolución de dudas y } \\
\text { organización } \\
(45 \text { min })\end{array}$ \\
\hline \multicolumn{4}{|c|}{ Día 6 (2h) } \\
\hline $\begin{array}{l}\text { Pregunta } \\
\text { relativa al mapa } \\
\text { de contenidos }\end{array}$ & & - & \\
\hline
\end{tabular}




\begin{tabular}{|c|c|c|c|}
\hline $\begin{array}{l}\text { Tipo de } \\
\text { actividad }\end{array}$ & Calentamiento inicial & $\begin{array}{c}\text { Trabajo autónomo por } \\
\text { parte del alumnado }\end{array}$ & Resolución de dudas \\
\hline $\begin{array}{l}\text { Referencia } \\
\text { al modelo } \\
\text { metodológico }\end{array}$ & & INVESTIGACIÓN & \\
\hline Descripción & $\begin{array}{c}\text { Se saluda, recapitula } \\
\text { lo del día anterior, se } \\
\text { prepara la clase para } \\
\text { comenzar el silencio } \\
\text { de clase. Se advierte } \\
\text { que hoy es el último } \\
\text { día de trabajo en } \\
\text { clase y que el trabajo } \\
\text { continuará en casa } \\
\text { (10 min) }\end{array}$ & $\begin{array}{c}\text { Los alumnos } \\
\text { comienzan a trabajar } \\
\text { en el proyecto } \\
\text { planteado. Hoy es } \\
\text { día de resolución de } \\
\text { dudas y organización } \\
\text { (90 min) }\end{array}$ & $\begin{array}{c}\text { Resolución de dudas y cuestiones } \\
\text { de cara a la exposición } \\
\text { (20min) }\end{array}$ \\
\hline \multicolumn{4}{|c|}{ Dia 7 (1h) } \\
\hline $\begin{array}{l}\text { Pregunta } \\
\text { relativa al mapa } \\
\text { de contenidos }\end{array}$ & & & Exploración final \\
\hline $\begin{array}{l}\text { Tipo de } \\
\text { actividad }\end{array}$ & $\begin{array}{l}\text { Preparación de las } \\
\text { exposiciones }\end{array}$ & $\begin{array}{l}\text { Realización del } \\
\text { cuestionario final }\end{array}$ & \\
\hline $\begin{array}{l}\text { Referencia } \\
\text { al modelo } \\
\text { metodológico }\end{array}$ & & $\begin{array}{l}\text { PRESENTACIÓN } \\
\text { EN CLASE } \\
\text { (resultado) }\end{array}$ & $\begin{array}{c}\text { Exploración } \\
\text { inicial }\end{array}$ \\
\hline Descripción & $\begin{array}{c}\text { Se recuerda la } \\
\text { dinámica de las } \\
\text { presentaciones y se } \\
\text { empieza a presentar } \\
\text { directamente para } \\
\text { no perder tiempo } \\
\text { y que de tiempo a } \\
\text { todos los grupos } \\
\text { (5 min) }\end{array}$ & $\begin{array}{l}\text { Se llevan a cabo las } \\
\text { presentaciones por } \\
\text { grupos } \\
\text { (95 min) }\end{array}$ & $\begin{array}{l}\text { Realización del cuestionario final y } \\
\text { reflexión en voz alta acerca de si ha } \\
\text { habido algún cambio con respecto } \\
\text { con el primer día } \\
(20 \mathrm{~min})\end{array}$ \\
\hline
\end{tabular}




\section{Aplicación del CIMA}

A continuación se expondrá el diario confeccionado por sesiones para facilitar la lectura y posterior evaluación del CIMA.

\section{Sesión 1}

Esta primera sesión se desarrolla de manera amena y sin complejidad. La profesora comienza recalcando la importancia de la innovación docente y lo significativas que serán las sesiones a partir de ahora para este bloque en concreto. Dado que son alumnos de primero de grado surgen algunas dudas y planteamientos acerca de las diferencias que encuentran entre la docencia universitaria y la de la educación secundaria. El debate deriva en una conversación acerca de las profesiones del futuro. Después de este calentamiento se comienza por repartir el cuestionario inicial que se desarrolla rápido y sin complejidad.

Una vez que acaba se comienza a plantear la teoría por ver de clase y se advierte del contenido eminentemente teórico que tendrá el resto de esta sesión. Se tratan conceptos como el de crisis, gestión de conflictos y parte del significado de la imagen corporativa. Para finalizar, dos voluntarios nos hacen un repaso de los apuntes sobre los que han tomado nota para hacer un cierre recordatorio de lo visto en clase.

\section{Sesión 2}

Esta sesión resulta mucho más amena que la anterior. En ella se llevan a cabo dos actividades que resultan muy gratificantes. Después de la actividad de calentamiento que se compone del saludo y de la recapitulación del día anterior, se comienza por la actividad relacionada con la 
búsqueda de las noticias de clase relacionadas con la crisis. En un primer momento sorprende que los alumnos se sienten poco seguros de lo que tienen que hacer. Dado que les ofrezco la posibilidad de utilizar el dispositivo móvil para llevar a cabo la búsqueda de noticias, parecen sentirse inseguros. Algunos aprovechan para consultar redes sociales y otro tipo de plataformas pero no le doy importancia ya que es algo que ocurre de forma puntual y casi todo el tiempo se encuentran muy activos en la participación y en el trabajo en clase. Se ve que hay implicación. Después de este tiempo, se explica que se llevará a cabo una escalera que ellos mismos tendrán que definir. Se tarda unos minutos en explicarla adecuadamente (más de lo esperado) pero luego el resultado, aunque inacabado porque no da tiempo a ver todas las noticias, es muy positivo.

La única objeción de esta sesión es que no se termina en el tiempo estimado ya que se ha requerido de un tiempo mayor para llevar a cabo las explicaciones. No da tiempo a colocar todas las noticias en la escalera ya que se detiene la exploración de noticias unos minutos antes para que de tiempo a hacer un comentario global de todas ellas y reflexionar sobre el sentido de la escalera y su vinculación con la materia teórica vista en clase.

\section{Sesión 3}

Esta sesión se abre con el saludo y calentamiento inicial para seguir con la teoría propuesta para el día. En esta ocasión se habla de la importancia de los medios de comunicación y se abre un pequeño paréntesis para recalcar las diferencias teóricas entre conceptos como "medio" y "canal" que de manera espontánea surgen a modo de duda entre los alumnos. A continuación se presenta el caso de crisis "Dodot", se les explica lo ocurrido y de manera también espontánea se abre un debate acerca de 
cómo proceder para resolver el caso. Se trata de un grupo muy abierto y participador que plantea muchas dudas y debates en clase. Se les explica la actividad que tienen que llevar a cabo acerca del dossier y se ponen a trabajar en ello.

\section{Sesión 4}

Esta sesión se inicia con el saludo diario y rápidamente el alumnado solicita la resolución de lo que llaman "dudas urgentes" que necesitan resolver para continuar con la actividad propuesta. Para responder a todos los grupos en la medida de los posible, la profesora se acerca mesa por mesa para reunirse con cada grupo y tratar de resolver las dudas. En un principio esta fase de la actividad en la que se pedía realizar una investigación y planteamiento del dossier no puede llevarse a cabo en el tiempo estimado porque el alumnado solicita un tiempo extra para acabar el dossier. Tras la deliberación de la profesora y la escucha de los grupos, se decide dejar el resto de la sesión y la siguiente para acabar el dossier.

\section{Sesión 5}

Dado que esta sesión se plantea para seguir trabajando y resolver dudas, se desarrolla sin mayor complejidad. Se resuelven dudas de manera individual que posteriormente se exponen en voz alta por parte de la profesora para que el resto sea consciente. Se cierra la sesión recordando que el próximo día comienzan las exposiciones.

Sesión 6

La clase comienza rápidamente para no perder tiempo de exposiciones y ajustarnos al tiempo estimado para este bloque de presentaciones. Se les recuerda a todos los 
alumnos cómo se llevarán a cabo las exposiciones y su dinámica. Se decide entre todos que el orden de exposiciones empezará por la letra $M$ de los apellidos del alumnado. Una vez que comienzan las exposiciones sorprende ver cómo presentan sus trabajos ya que se destaca cierta seguridad y serenidad (en la mayoría de grupos) a la hora de exponer sus ideas. Resulta gratificante verles contar cómo han trabajado y comprobar que los resultados son muy óptimos.

\section{Sesión 7}

La clase comienza directamente con la puesta en común de los dossieres de cada grupo que quedaban por exponer. Se cierra esta parte de la sesión comentado por encima los aspectos positivos de las exposiciones en general y las debilidades encontradas. Para acabar el día se realiza el cuestionario final. Una vez finaliza se lleva a cabo un debate entre todos acerca de si se han visto diferencias entre los cuestionarios finales y los iniciales. En general, es muy satisfactorio ver cómo de manera generalizada se observan cambios. Se discute acerca de la experiencia y se recogen las apreciaciones de los alumnos.

\section{Evaluación del CIMA y principios didácticos}

Este CIMA ha contado con dos instrumentos de evaluación principales. En primer lugar, se ha empleado una escalera de aprendizaje para la actividad anteriormente explicada acerca de la colocación de las noticias y que ha servido como ejercicio exploratorio y, por otro, una rúbrica para evaluar el dossier elaborado y presentado en las exposiciones de las últimas sesiones.

Como se observa en la Figura 3, se han identificado cuatro grandes bloques por parte del alumnado para 
identificar el tipo de noticias encontradas y su vinculación con la teoría vista en clase acerca de la gestión de crisis. En este sentido, los bloques acordados por el alumnado se reconocen muy alejados de la teoría y conceptos a los que se quiere llegar en las futuras sesiones. El alumnado destaca el carácter de la noticia teniendo como referencia el propio contenido y no haciendo una reflexión que implique las posibles consecuencias que la noticia pudiera tener para la empresa. Se aplican evaluaciones de las noticias del tipo "son noticias falsas", "son dañinas", o, "son negativas". Si bien es cierto que es una apreciación relativamente normal para el grado de conocimiento de RR.PP de un primer grado en publicidad, sí se destaca que el vocabulario empleado es poco riguroso. Cabe señalar que hay algunos alumnos (procedentes de ciclos formativos) que sí diferencian las noticias como potencialmente problemáticas para la empresa y que pudieran dar lugar a crisis futuras.

\section{¿Cómo evaluarias esta noticia?}

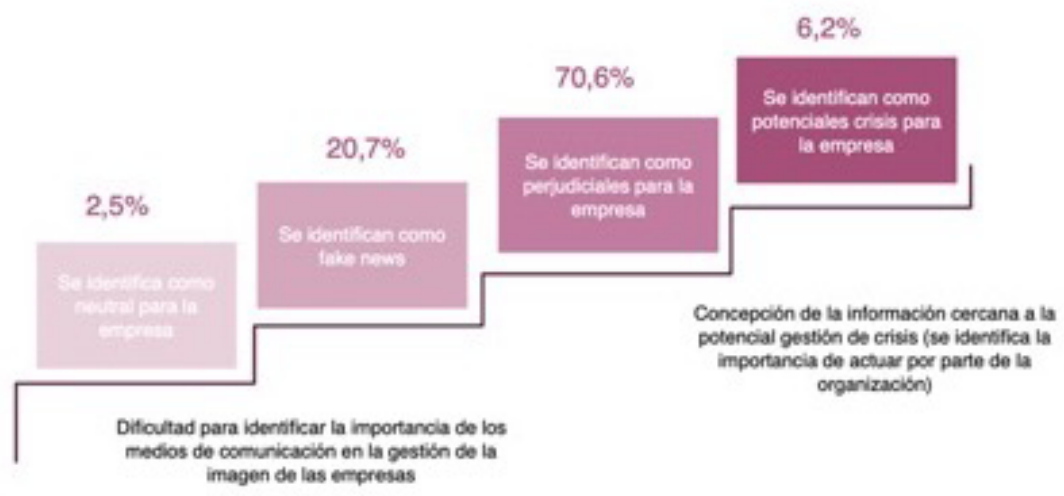

Figura 3. Escalera de aprendizaje comparativa.

Otro de los instrumentos de evaluación empleados ha sido la rúbrica para evaluar el dossier elaborado por parte del alumnado. Este dossier se presenta como un 
documento de gran utilidad para llevar a cabo la presentación final. Se trata de elaborar un documento en el que el alumno tiene que idear un plan de crisis para dar solución al problema de la organización planteada, en este caso, la empresa Dodot.

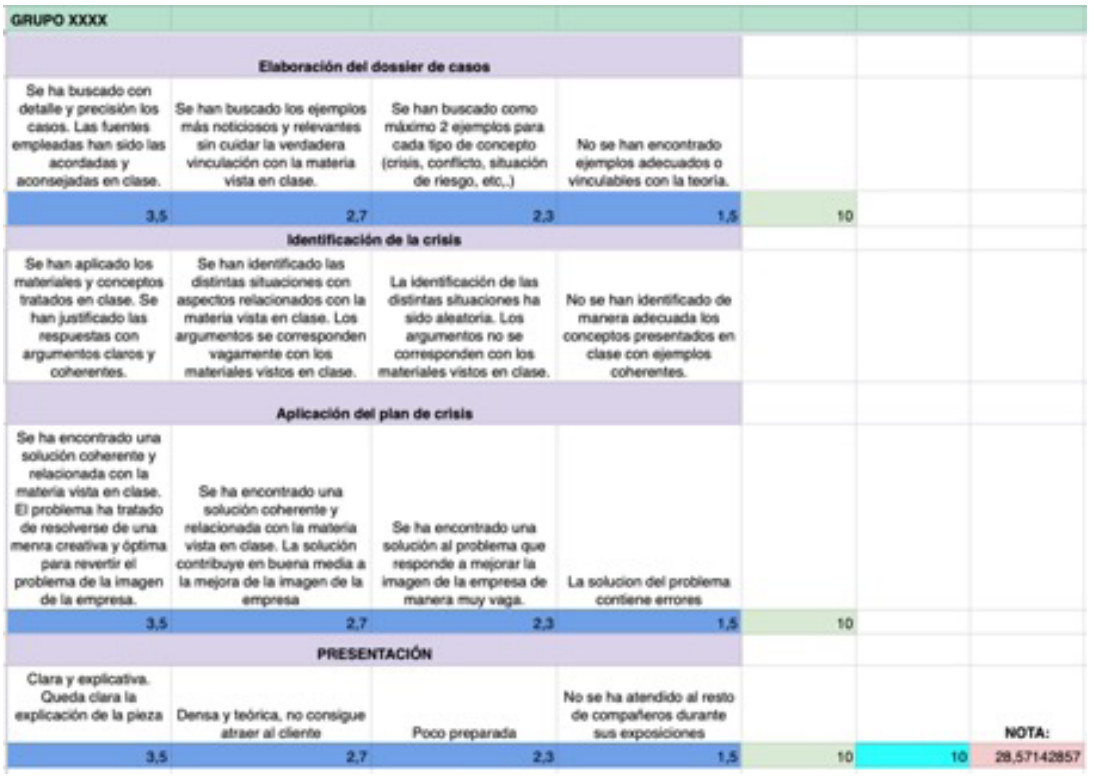

Figura 4. Rúbrica de evaluación para el Dossier de gestión de Crisis.

Si atendemos a la Figura 4, la rúbrica se compone de cuatro ítems a valorar con respecto a la entrega del dossier y su exposición; la elaboración de casos, la identificación de la crisis, la aplicación del plan y, por último, la presentación. A su vez, cada ítem se corresponde con diferentes evaluaciones que van acompañadas de las distintas puntuaciones. Esta rúbrica no solo es significativa desde el punto de vista del profesor para llevar a cabo la evaluación, al mismo tiempo, se trata de una herramienta muy útil para el alumnado ya que le permite conocer desde el inicio de la práctica los ítems a valorar. En definitiva, estas herramientas pretenden contribuir al aprendizaje útil y significativo del alumnado y se presentan como mecanismos útiles para la evaluación del mismo. En cuanto a 
los principios didácticos que se han considerado para este CIMA, se ha observado, en primer lugar, que la planificación previa y estudio del alumnado se hacen claves para llevar a cabo una actividad de innovación docente. Dedicar tiempo a una correcta planificación teniendo en cuenta las necesidades y el contexto del alumnado, ofrece un primer paso para implementar con éxito actividades innovadoras que requieren de una alta implicación por parte de los alumnos. En este sentido, se reflexiona acerca de la necesidad de mantener como principio didáctico actividades de evaluación inicial para conocer el estado de los alumnos y planificar en consecuencia las actividades futuras.

Asimismo, se destaca la importancia de la evaluación de tipo "compartido", es decir, aquella evaluación que se presenta al inicio de cualquier actividad y que se conoce al detalle por parte del alumnado. En este sentido, se plantea que para un futuro este tipo de evaluaciones en formato rúbricas podrían funcionar de manera pactada, esto es, negociando con el alumnado aquellos ítems que se deben de evaluar y definir en conjunto cómo deben de ser valorados.

Jornadas de Formación e Innovación Docente del Profesorado | № 2 (2019) Esta obra se distribuye con la licencia Creative Commons 


\section{Referencias}

Bain, K. (2004). Lo que hacen los mejores profesores universitarios. Valencia: Universita de Valencia.

Finkel, D. (2008). Dar clase con la boca cerrada. Valencia: Universitat de Valencia.

Porlán, R. (Coord.). (2017). Enseñanza universitaria. Cómo mejorarla. Madrid: Ediciones

Morata.

Jornadas de Formación e Innovación Docente del Profesorado | № 2 (2019) Esta obra se distribuye con la licencia Creative Commons 